ARTIGO DE ATUALIZAÇÃO

ROCHELE PAZ FONSECA

GIOVANA COGHETTO SGANZERLA

LARISSA VALENCY ENÉAS

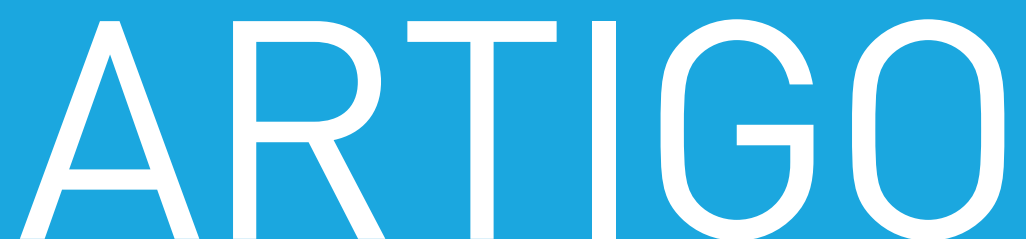

\title{
FECHAMENTO DAS ESCOLAS NA PANDEMIA DE COVID-19: IMPACTO SOCIOEMOCIONAL, COGNITIVO E DE APRENDIZAGEM
}

\section{SCHOOL CLOSURE IN THE COVID-19 PANDEMIC: SOCIOEMOTIONAL, COGNITIVE AND LEARNING IMPACT}

\begin{abstract}
Resumo
A pandemia de COVID-19 acarretou, em nível mundial, o fechamento das escolas poratéaproximadamente 8 meses em 2020. Um dos períodos mais longos de afastamento de crianças e adolescentes da aprendizagem presencial e da convivência social ocorreu e ainda ocorre no Brasil. Embora não se possa negar que muitas características do vírus e de suas consequências sejam ainda pouco conhecidas, as evidências e estimativas até o momento embasam um posicionamento a favor da abertura das escolas, com todos os cuidados recomendados por órgãos científicos e representativos da saúde e da educação. Este artigo visa mapear evidências e documentos científicos com interpretações da neuropsicologia e da medicina acerca do impacto individual e coletivo socioemocional, cognitivo e de aprendizagem acadêmica do fechamento das escolas durante a pandemia. Crianças, quanto mais jovens forem, tendem a se desenvolver muito em poucos meses, considerando-se evidências neurocientíficas das janelas ótimas de desenvolvimento. A convivência social e a formação/consolidação de hábitos de aprendizagem socioemocional, de leitura e estudos oportunizadas no ambiente escolar são únicas. Três argumentos-chave são abordados e sinteticamente analisados no presente artigo: 1) os indícios sobre transmissibilidade e epidemiologia da COVID-19 em crianças e familiares/professores a elas relacionados alicerçam relativa segurança de que haja menos riscos e mais benefícios; 2) os prejuízos para o desenvolvimento cognitivo, socioemocional e de aprendizagem escolar propriamente dita para estudantes já estão sendo fortemente evidenciados, além das estimativas de impactos geracionais de longo prazo a
\end{abstract}

serem observados por pelo menos quatro décadas; e 3) a saúde mental e o custo de trabalho posterior para pais, professores e instituições escolares encontram-se em zona de risco. Por fim, além de evidências, reflexões finais que respeitam que o cerne desta análise está sob um campo de decisão individual são abordadas, contrapondo-se também à relevância de se considerar o impacto coletivo para o desenvolvimento de uma geração e de uma sociedade com 1 ano ou mais de vivência escolar interrompida, principalmente no contexto da vulnerabilidade sociocultural, econômica, emocional e cognitiva.

Palavras-chave: Fechamento das escolas, isolamento social, COVID-19, aprendizagem, desenvolvimento cognitivo, desenvolvimento socioemocional.

\section{Abstract}

The COVID-19 pandemic resulted in the closure of schools worldwide for up to approximately eight months in 2020. One of the longest periods of removal of children and adolescents from face-to-face learning and social coexistence has occurred and still occurs in Brazil. Although it cannot be denied that many characteristics of the virus and its consequences are still poorly known, the evidence and estimates so far support a position in favor of opening schools, following all the precautions recommended by scientific institutions and health and education authorities. This article aims to map evidence and scientific documents with interpretations, from the point of view of neuropsychology and medicine, of the individual and collective impact of school closure on the socioemotional, cognitive and academic learning arenas. 


\section{ROCHELE PAZ FONSECA ${ }^{1}$}

1 Professora titular, Faculdade de Psicologia e Programa de Pós-Graduação em Psicologia (Cognição Humana), Pontifícia Universidade Católica do Rio Grande do Sul (PUCRS), Porto Alegre, RS. Presidente, Sociedade Brasileira de Neuropsicologia (SBNp), São Paulo, SP. ${ }^{2}$ Psicóloga pela PUCRS, Porto Alegre, RS. Pós-graduanda em Psicologia do Desenvolvimento e da Aprendizagem, PUCRS, Porto Alegre, RS. ${ }^{3}$ Graduação em Medicina, Universidade Federal do Rio Grande do Sul (UFRGS), Porto Alegre, RS. Residência Médica em Otorrinolaringologia, Hospital São Lucas da PUCRS, Porto Alegre, RS. Fellowship em Otorrinolaringologia Pediátrica, Hospital de Clínicas de Porto Alegre(HCPA), Porto Alegre, RS. Mestre em Saúde da Criança e Adolescente pela UFRGS, Porto Alegre, RS.

Children, especially the younger ones, tend to develop a lot in a few months, considering neuroscientific evidence of optimal developmental windows. Social coexistence and the formation/consolidation of socioemotional learning habits, reading habits and study opportunities in the school environment are unique. Three key arguments are addressed and briefly analyzed in this article: 1) the evidence on transmissibility and epidemiology of COVID-19 in children and their relatives/teachers support the notion that the benefits of school opening outweigh the risks; 2) there has been strong evidence of damage to cognitive, socioemotional and school learning development for students, in addition to the estimate of long-term generational impacts to be observed for at least four decades; and 3) the mental health and cost of subsequent work for parents, teachers and school institutions seem to be another risk zone. Finally, in addition to evidence, final reflections are made taking into consideration that the core of this analysis is a matter of individual decision, considering the opposing force of the collective impact of a year or more of interrupted school experience on the development of a generation and a society, especially in the context of sociocultural, economic, emotional and cognitive vulnerability.

Keywords: School closure, social isolation, COVID19, learning, cognitive development, socioemotional development.

\section{INTRODUÇÃO}

O tema abordado neste artigo é ousado e polêmico; porém, é indubitavelmente necessário e oportuno: como decidir pela volta às aulas ou pela manutenção do fechamento de instituições educacionais até a vacinação? $\bigcirc$ que colocar na balança para uma tomada de decisão, considerando-se riscos e benefícios da convivência escolar e da aprendizagem acadêmica para o desenvolvimento socioemocional, cognitivo, de conteúdo em nível individual e coletivo? Neste artigo de atualização, apresentar-se-á uma revisão sintética, mas suficiente para embasar uma reflexão acerca desta desafiadora decisão, visando-se à defesa argumentativa e crítico-analítica de um posicionamento pró-retorno às escolas.

A pandemia de COVID-19 foi considerada um caso de emergência de saúde pública mundial e obrigou diversos países a adotarem medidas rigorosas de restrição de mobilidade e de distanciamento social ${ }^{1,2}$. Estima-se que 137 países fecharam as escolas como parte da política de distanciamento procurando conter a transmissão do SARS-COV-2 ${ }^{3}$. O fechamento das escolas por aproximadamente 8 meses em 2020 acabou por caracterizar-se como um dos períodos mais longos de afastamento de crianças e adolescentes da aprendizagem presencial e da convivência social que ocorreu e ainda ocorre no Brasil.

Embora não se possa negar que muitas características do vírus e de suas consequências sejam ainda pouco conhecidas, as evidências e estimativas até o momento embasam um posicionamento a favor da abertura das escolas, com todos os cuidados recomendados por órgãos científicos e representativos da saúde e da educação. Neste artigo, inspirado em duas aulas abertas promovidas pela primeira autora, Rochele Paz Fonseca, representando a visão de sua formação e estudos contínuos em psicologia, fonoaudiologia e da neuropsicologia, juntamente com a última autora, Larissa Valency Enéas, representando a formação e estudos atuais da medicina e da otorrinopediatria, espera-se contribuir para que posicionamentos polarizados ou dicotomizados não se embasem em falta de informação ou em viés de transmissão de conteúdo resultando em um quase "terrorismo psicológico".

Este artigo visa mapear evidências e documentos científicos com interpretações da neuropsicologia e da medicina acerca do impacto individual e coletivo socioemocional, cognitivo e deaprendizagemacadêmica do fechamento das escolas durantea pandemia, sem quaisquer objetivos políticos. Espera-se poder contribuir, com base na leitura de mais de 100 artigos científicos e documentos de órgãos representativos, para o planejamento de ações de políticas públicas pró-aprendizagem, desenvolvimento acadêmico, revalorização dos professores e da instituição escolar em nossa sociedade. Dados e argumentos de três vertentes serão apresentados para uma discussão, que para alguns pode ser final (pró-decisão de abrir escolas ou de levar seus filhos de volta a ela), ou, dependendo do ângulo de análise, inicial, respeitando-se a complexidade do tema e da tomada de decisão coletiva de uma nação, de um estado ou de um município, como individual de uma família. 


\section{ROCHELE PAZ FONSECA}

GIOVANA COGHETTO SGANZERLA

LARISSA VALENCY ENÉAS

\section{EVIDÊNCIAS DE SAÚDE FÍSICA, MENTAL E DE APRENDIZAGEM}

Vertente 1: os indícios até o momento de estudos sobre transmissibilidade e epidemiologia em crianças e familiares/professores a elas relacionados alicerçam relativa segurança de que haja menos riscos e mais benefícios

O fechamento das escolas resultou de uma tentativa inicial de tentar conter a propagação de um vírus pouco conhecido até aquele momento. Com os avanços nas pesquisas acerca da transmissão e medidas de prevenção, constatou-se que o ambiente escolar não é um super disseminador (super spreader) e que, mantidas as medidas de biossegurança necessárias, pode-se defender a sua reabertura. Embora as evidências sejam ainda limitadas, a maioria dos estudos sugere que crianças nãosão potenciais transmissoras do vírus, indicando que tenham uma menor probabilidade de serem infectadas ${ }^{4-6}$. Além disso, as taxas de contaminação em ambientes educacionais são relativamente baixas ${ }^{1}$. Sabe-se, portanto, que crianças parecem ter menor suscetibilidade à infecção quando comparadas a adultos e que, quando infectadas, geralmente são oligossintomáticas (apresentam sintomas leves) ou assintomáticas ${ }^{5,7,8}$. Isso pode ser explicado pela estimativa de que crianças têm uma expressão menor de ACE2, que são enzimas receptoras do vírus nas células humanas. Portanto, possuem risco menor de infecção e que, por algum mecanismo ainda não bem compreendido, estão mais bem protegidas de possível agravo da doença, caso a contraiam?

Discute-se, também, sobre o grau de letalidade em crianças com comorbidades que, apesar dos poucos dados disponíveis, sabe-se que podem se tornar agravantes do quadro clínico em caso de contaminação $\mathrm{e}$, neste caso, orienta-se que continuem em isolamento social ${ }^{10}$. Em um estudo que investigou o grau de risco de contágio em crianças na escola e em casa, foi identificada uma diferença insignificante em comparação ao efeito geral do fechamento das escolas, visto que o contato reduzido na escola leva a um maior contato com os familiares ${ }^{11}$. Não está claramente documentado por que a transmissão de criança-criança e criança-adulto é tão infrequente.

Em outros países, os achados também sugerem a possibilidade de volta às aulas. Na Inglaterra, as taxas de infecção e transmissão foram baixas tanto na pré-
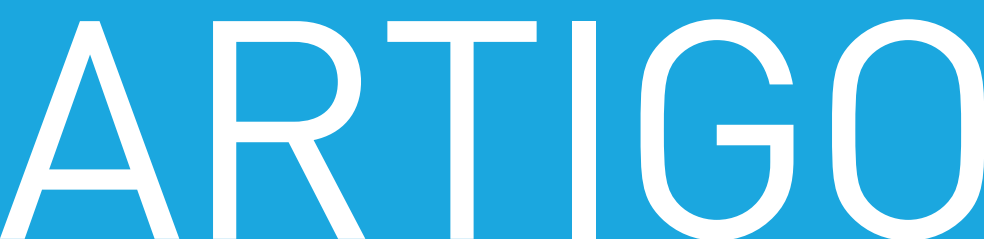

escola como nas escolas primárias, assim como entre funcionários. Nessa pesquisa, foram monitoradas 131 escolas, em que 12.047 participantes foram testados. Os achados mostraram que a taxa de infecção entre os alunos foi de 3,9 a cada 100.000 por semana; enquanto que, para funcionários, de 11,3 para 100.000 por semana ${ }^{12}$. Na França, outro estudo também mostrou que existe baixa probabilidade de infecção secundária entre crianças $^{13}$. $\mathrm{Na}$ Suíça, um estudo envolvendo 39 famílias constatou que em apenas três (8\%) havia um caso suspeito, com início dos sintomas após ou concomitantemente aos adultos, o que sugere a maior frequência de contaminação por adultos. A China, por sua vez, apresentou resultados de investigações de transmissão doméstica, em que, das 68 crianças com COVID-19 internadas no hospital e que fizeram parte dos estudos, 65 (ou seja, 95,59\%) eram pacientes provenientes de contatos domésticos adultos previamente infectados ${ }^{5,14}$. Esses achados permitem refletir sobre a extensão da real responsabilidade das crianças na transmissão do coronavírus.

Entretanto, há a necessidade de cautela ao analisar outros achados de associação mais forte, como no estudo de Auger et al. ${ }^{15}$, visto que outras estratégias de distanciamento foram tomadas, como o fechamento de serviços não essenciais e pedido de permanência em casa. Ainda nesse sentido, este estudo não discute e analisa os mecanismos pelos quais o fechamento das escolas pode afetar na transmissão viral do SARS-CoV-2. A análise não diferencia a duração, combinação e sequência ideal de intervenção de distanciamento, como o fechamento das escolas. Deve-se ponderar outros aspectos que impactam na vida dos estudantes, como as possíveis consequências acadêmicas, de saúde e econômicas, pois essas variáveis apresentam um continuum mais horizontal em longo prazo, sendo capaz de apresentar consequências que podem perdurar décadas, além da difícil mensuração atual16.

Dados mais recentes sugerem que o fechamento das escolas não contribuiu e não contribui para o controle da pandemia ${ }^{17}$, visto que a medida parece não se aplicar à SARS-CoV-2 pela dinâmica de transmissão do vírus ${ }^{18}$. Ademais, o fechamento emergencial foi precoce, na medida em que se baseou em estimativas a partir de epidemias anteriores, especialmente causadas pelo vírus influenza, em que as crianças eram os principais 


\section{ROCHELE PAZ FONSECA ${ }^{1}$}

1 Professora titular, Faculdade de Psicologia e Programa de Pós-Graduação em Psicologia (Cognição Humana), Pontifícia Universidade Católica do Rio Grande do Sul (PUCRS), Porto Alegre, RS. Presidente, Sociedade Brasileira de Neuropsicologia (SBNp), São Paulo, SP. ${ }^{2}$ Psicóloga pela PUCRS, Porto Alegre, RS. Pós-graduanda em Psicologia do Desenvolvimento e da Aprendizagem, PUCRS, Porto Alegre, RS. ${ }^{3}$ Graduação em Medicina, Universidade Federal do Rio Grande do Sul (UFRGS), Porto Alegre, RS. Residência Médica em Otorrinolaringologia, Hospital São Lucas da PUCRS, Porto Alegre, RS. Fellowship em Otorrinolaringologia Pediátrica, Hospital de Clínicas de Porto Alegre(HCPA), Porto Alegre, RS. Mestre em Saúde da Criança e Adolescente pela UFRGS, Porto Alegre, RS.

transmissores ${ }^{4}$. Uma pesquisa que estudou os efeitos do fechamento das escolas alerta para o fato de que as estratégias para redução da mortalidade por COVID-19 são diferentes das que visam reduzir a ocupação de UTI pelo mesmo motivo e das que reduzem o número de casos. O estudo, portanto, declara ser impossível otimizar uma estratégia de combate ao vírus, a menos que se priorize cada um dos resultados desejáveis ${ }^{11}$. Dessa forma, a retomada das atividades escolares presenciais parece um dos principais recursos para minimizar sequelas até o desenvolvimento e a distribuição de uma vacina ou até que se atinja melhores taxas de imunidade coletiva.

Vertente 2: os prejuízos para o desenvolvimento cognitivo, socioemocional e de aprendizagem escolar propriamente dita para estudantes já estão sendo fortemente evidenciados, além das estimativas de impactos geracionais de longo prazo a serem observados por pelo menos quatro décadas

A saúde mental é surpreendentemente ainda negligenciada na ciência e nas políticas públicas, o que se evidenciou no expressivo número de publicações desde março, após o começo da maior pandemia de COVID-19, que abordaram critérios, objetivos e fatores de ponderação para diretrizes predominantemente representativos da saúde física ${ }^{19,20}$. Além da importância da saúde mental em si, é crucial a retomada da conscientização da relação entre saúde mental e física, até mesmo no que tange aos aspectos imunológicos. Há inúmeras evidências indicando que quanto melhor a saúde mental, melhor a imunidade ${ }^{21-24}$

O momento atual suscita cuidados especiais em relação à saúde mental das crianças e adolescentes, tendo em vista que se encontram em um período importante do desenvolvimento. No que se refere à qualidade da aprendizagem, é preciso atenção, já que alguns estudos sugerem menor eficácia do ensino à distância com essas faixas etárias. De tal forma, o acompanhamento dos pais na rotina da criança é imprescindível ${ }^{20,25}$. Além disso, pelo fato de o estudante passar a maior parte do tempo em casa, os pesquisadores alertam para o possível ganho de peso, maior uso de tempo de tela e alterações no padrão de sono 20,25 .

Primeiramente, o impacto da educação remota ou online em caráter emergencial não deve ser interpretado de modo linear nas diferentes faixas etárias, havendo clusters de desenvolvimento cognitivo e socioemocional infantil versus fase escolar. Crianças de 0 a 3 anos de idade (subgrupo pré-escolar 1) e de 4 até 6 anos (subgrupo pré-escolar 2) encontram-se na etapa de educação infantil, em que estão desenvolvendo, pelo essencial papel do brincar e da gradativa maior exposição a tarefas sistematizadas pré-leitura, préescrita e pré-matemática, sua prontidão escolar, em inglês conhecida como school readiness e muito estudada. Entre as principais condições a serem desenvolvidas para que a criança ingresse no ensino fundamental e se alfabetize para uma aprendizagem acadêmica e um desenvolvimento socioemocional suficiente e saudável preparatório para a vida futura, destacam-se motricidade ampla e fina (para escrita), autorregulação emocional e funções executivas (sustentação atencional, automonitoramento demotivaçãointrínseca, postergação de ganhos ou adiamento de recompensas), linguagem oral suficientemente desenvolvida quanto à consciência fonológica, ao vocabulário e ao processamento de narrativas, conhecimento básico de diferenciação de letras e números com noções de quantificação, entre outras $^{25}$. Nesse período pré-escolar, o desenvolvimento ocorre de modo muito acelerado e interaçãodependente. Assim, 7 meses de afastamento da préescola, para uma criança de 0 a 3 anos de idade, pode significar um sexto ou mais da sua vida com privação de estimulação da convivência com pares. Adiciona-se a essa estimativa o conhecido papel da assistência à pré-escola para o desenvolvimento global do indivíduo, como o comprovado para o desenvolvimento de numeracia e habilidades matemáticas descrito no estudo de Aunio et al. ${ }^{26}$.

O segundo cluster compõe-se pelas crianças no primeiro e segundo anos do ensino fundamental, em fase de aquisição e consolidação preliminar, mas essencial, de leitura, escrita e matemática. O ensino sistemático e dirigido, mediado por motivação, ludicidade e modelagem do grupo, é o alicerce principal. Posteriormente, poderiam-se agrupar as crianças do ensino fundamental I (do terceiro ao quinto ano), do ensino fundamental || e os adolescentes do ensino médio. Quanto mais velha a criança, melhor tende a ser o desenvolvimento da autonomia, da independência e da funcionalidade para 


\section{ROCHELE PAZ FONSECA}

GIOVANA COGHETTO SGANZERLA

LARISSA VALENCY ENÉAS

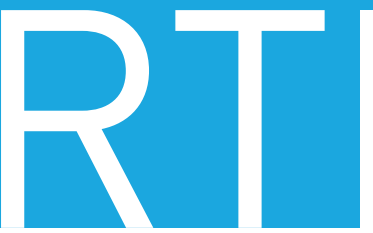

GO estudar mediante hábitos e estratégias automonitorados. No entanto, mesmo na adolescência, sabe-se que há um período muito crítico e crucial de desenvolvimento neurobiológico de habilidades cognitivas mais complexas que dependem da interação ambiental ${ }^{27}$.

Estudos demonstram que períodos longe da escola podem impactar em aspectos de saúde mental e processo de aprendizagem das crianças, como os achados que afirmam que o período de férias também está associado a um prejuízo na saúde emocional e cognitiva, além de bem-estar na vida de crianças e adolescentes $^{28}$. Entretanto, apesar de a pandemia de COVID-19 exigir novas adaptações, isto é, apesar do distanciamento social, o processo de aprendizagem continuou digitalmente. O questionamento focaliza-se, nesse âmbito, sobre as limitações de atenção, motivação e memorização da aprendizagem na ausência de uma mediação docente presencial e da modelagem dos pares em um grupo com sensação de universalidade.

Mais especificamente, no contexto da vulnerabilidade socioeconômica e cultural, sabe-se que o fechamento das escolas pode aumentar a lacuna de aprendizagem entre as crianças de menor a maior poder aquisitivo, principalmente pelo fato de que o ensino digital exige condições mínimas de conexão de internet e materiais eletrônicos que deem acesso aos sistemas escolares. Um estudo na Europa encontrou que 5\% de crianças não vivem em uma casa adequada para poder estudar, e 6,9\% não possuem acesso à internet ${ }^{29}$. No Brasil, essa discrepância é ainda mais gritante e limitante.

Em estudos pré-pandêmicos, a disparidade de desenvolvimento cognitivo e de desempenho escolar já era evidenciada em países em desenvolvimento como o Brasil ${ }^{30,31}$. O que tende a acontecer no retorno às aulas em 2021, quando o conteúdo do próximo ano deveria se alicerçar em uma mínima, mas suficiente consolidação da aprendizagem do conteúdo de 2020, que foi ministrado à distância, por atividades remotas em sua maioria? A integração de conhecimentos tende a ficar comprometida face às limitações dos recursos virtuais. De tal forma, o gap já pré-existente provavelmente aumentará, o que conduzirá a um efeito "bola de neve" muito danoso ao desenvolvimento multidimensional desses indivíduos aprendizes, pelo fenômeno de economia cognitiva. Esse último acarreta uma espécie de desligamento da pró-atividade mental para aprender estímulos e episódios inalcançáveis, por seu nível de complexidade e pela falta de conexões prévias, que oportunizem uma aprendizagem integrada, significativa e duradoura.

Em suma, mais de $90 \%$ de crianças e adolescentes no mundo inteiro ficaram ou ainda estão afastados da escola por um período até maior em distanciamento social. O confinamento em si levou ao cancelamento temporário de quaisquer outras atividades de lazer, de convivência e esportivas fora de casa ${ }^{32}$. Questionase: as crianças e os adolescentes se recuperarão facilmente? Hipotetiza-se que somente aqueles que tiverem maior resiliência socioemocional e cognitiva, menores índices ou níveis de gravidade de doença mental e de quadros neurodesenvolvimentais, com mais fatores de risco para os estudantes do contexto público. Assim, em todos os clusters de diferentes faixas etárias e de ciclos educacionais, são evidenciadas consequências que podem ilusoriamente ser julgadas em curto prazo como ausentes, caso tenham elevada resiliência e neuroplasticidade infantil; no entanto, há estimativas de sequelas em médio e longo prazos que poderão ser observadas em até uma década futura.

O período de isolamento social, necessário em um primeiro momento, caracterizou-se por vários fatores estressores para as crianças, tais como manejo do tédio ou da monotonia, desapontamento, falta de contato face a face com colegas, amigos e professores, espaço eventualmente mais reduzido em casa, com diminuição do gasto energético físico-motor, além da maior demanda de gerenciamento e de autorregulação de novos sentimentos delas mesmas e de seus pais, com perdas financeiras e laborais durante o lockdown ${ }^{19}$.

Emcidadesnorte-americanas, cujas pessoas presenciam tempestades de neve intensas, o fechamento das escolas já demonstrou afetar o aprendizado das crianças, assim como sua rotina diária, dos pais, professores e demais funcionários da instituição. Estas intempéries podem durar alguns dias ou semanas e, mesmo que por um curto período de tempo, as crianças têm apresentado pior desempenho nos testes relacionados ao processo de aprendizagem ${ }^{33}$, o que serve de embasamento para pensarmos nos prejuízos atrelados às restrições vividas pelas crianças na pandemia. 


\section{ROCHELE PAZ FONSECA ${ }^{1}$

1 Professora titular, Faculdade de Psicologia e Programa de Pós-Graduação em Psicologia (Cognição Humana), Pontifícia Universidade Católica do Rio Grande do Sul (PUCRS), Porto Alegre, RS. Presidente, Sociedade Brasileira de Neuropsicologia (SBNp), São Paulo, SP. ${ }^{2}$ Psicóloga pela PUCRS, Porto Alegre, RS. Pós-graduanda em Psicologia do Desenvolvimento e da Aprendizagem, PUCRS, Porto Alegre, RS. ${ }^{3}$ Graduação em Medicina, Universidade Federal do Rio Grande do Sul (UFRGS), Porto Alegre, RS. Residência Médica em Otorrinolaringologia, Hospital São Lucas da PUCRS, Porto Alegre, RS. Fellowship em Otorrinolaringologia Pediátrica, Hospital de Clínicas de Porto Alegre(HCPA), Porto Alegre, RS. Mestre em Saúde da Criança e Adolescente pela UFRGS, Porto Alegre, RS.

Vertente 3: a saúde mental e o custo de trabalho posterior para pais, professores e instituições escolares encontram-se em zona de risco

Os pais, desde o início da pandemia, foram obrigados, em sua maioria, a tentar enfrentar do melhor modo possível, contando com suas estratégias de resiliência e de coping, as adaptações do trabalho em home office com crianças e adolescentes em casa, necessidade de suporte a pais idosos ou outros familiares, pressão econômica, redução de espaço, entre outros ajustes emergenciais. Com isso, o risco de vulnerabilidade de sua saúde mental aumentou consideravelmente, com dados de mais violência doméstica, por exemplo ${ }^{32,33}$. Em um estudo com 183 pais de crianças nos EUA, fatores estressores relacionados ao COVID-19, como os elevados índices de ansiedade e de sintomas depressivos, mostraram-se associados à autopercepção parental de estresse, assim como a um maior potencial de abuso infantili ${ }^{34}$.

A partir de uma breve revisão promovida por Brooks et al. ${ }^{35}$, publicada também na The Lancet, incluindo 24 estudos sobre efeitos de diferentes quarentenas desde 2003, os achados mais prevalentes foram de efeitos psicológicos negativos, como de sintomas de estresse pós-traumático (um quadro descoberto em períodos peri e pós-guerras), desorientação ou confusão, episódios de raiva, com estimativa de consequências duradouras, persistentes. Há, ainda, relatos de aumento da dependência parental e de habilidades reduzidas de autonomia e de independência ${ }^{36}$.

\section{O QUE FAZER E COMO VOLTAR CASO A DECISÃo COLETIVA E/ OU INDIVIDUAL SEJA PRÓ-RETORNO ÀS AULAS?}

Cabe ressaltar que a reabertura das instituições escolares depende, também, do controle local da COVID19, para que se possa desenvolver estratégias seguras e de menor risco possível à comunidade escolar. Reabrir as escolas não significa voltar ao normal, mas considerar a necessidade do convívio social e da reinserção em um ambiente de aprendizagem em prol do desenvolvimento cognitivo e da saúde mental das crianças, avaliandose os benefícios e efeitos adversos das medidas aqui discutidas.

Muitos países passaram a adotar medidas parciais, que incluem a restrição do tamanho das turmas; aulas com horários escalonados; divisão e alternância de grupos de alunos entre aulas presenciais e remotas; e retorno à escola apenas para grupos de idade específicos². Ademais, é recomendado: isolar estudantes, professores e demais funcionários doentes ou com suspeita de contaminação; reforçar a higiene através da aplicação da lavagem regular das mãos e uso de álcool em gel, disponibilizado nas entradas/saídas; limpar diariamente superfícies; ventilar adequadamente os ambientes; e promover sempre que possível distanciamento social ${ }^{37,38}$. A Organização Mundial da Saúde (OMS) e o Fundo das Nações Unidas para a Infância ${ }^{37}$ aconselham o uso de máscara como método de intervenção de saúde pública que pode ajudar a prevenir e controlar a transmissão de vírus respiratórios, como o SARS-COV-2, principalmente a partir de 6 anos de idade ${ }^{38}$.

Em suma, na Tabela 1, apresentam-se as descrições sintetizadas dos quatro principais documentos que podem ser acessados e consultados por pais, gestores escolares e membros da sociedade geral e científica.

\section{CONSIDERAÇ̃̃ES FINAIS}

As notícias nos primeiros meses foram muito aterrorizantes e ainda embasadas na premissa temporária de que não se sabia nada acerca do vírus. Atualmente, evidências já estão disponíveis mostrando uma menor transmissibilidade entre crianças e destas para adultos. Considerando-se a educação como um pilar básico da sociedade, assim como a saúde mental e física, justamente pelo fato de a primeira ser promotora das últimas e fator de prevenção de doenças, os professores e gestores educacionais devem ser valorizados pela sociedade geral e, consequentemente, por políticas públicas, pois são também profissionais de linha de frente.

Além de evidências, reflexões finais que respeitam que o cerne desta análise está sob um campo de decisão individual são consideradas. Deve-se considerar os valores familiares e as condições de maior medo de cada indivíduo, família ou grupo social, assim como os índices de contaminação de cada cidade. Em complementaridade, não se pode negligenciar a relevância de se considerar o impacto coletivo para o desenvolvimento de uma geração e de uma sociedade com 1 ano ou mais de vivência escolar interrompida, principalmente no contexto da vulnerabilidade sociocultural, econômica, emocional e cognitiva. Consequências socioemocionais, cognitivas e 
Tabela 1 - Descrição de quatro documentos-chave acerca das consequências para a saúde mental e desenvolvimento infantil no fechamento das escolas

\begin{tabular}{lll}
\hline Documento & Data de publicação & Objetivo \\
\hline The future is here & Agosto/2020 & Apresentar a \\
(United Nations) $^{39}$ & & campanha "Salve \\
& nosso futuro" \\
& e alertar para a \\
& importância da \\
& reabertura das \\
& escolas.
\end{tabular}

Key Messages and Actions for COVID19 Prevention and Control in Schools $\left(\right.$ UNICEF) ${ }^{37}$
Março/2020

COVID-19 in children and the role of school settings in COVID19 (ECDC) ${ }^{2}$
Agosto/2020

Abordar o papel da escola na transmissão de COVID-19, apresentando dados sobre o comportamento do vírus em crianças.

\section{Principal conteúdo}

António Guterres, atual Secretário Geral da ONU, chama a atenção para os principais motivos que o levam a defender a reabertura das escolas. Entre eles, ressalta a importância da educação como chave para um desenvolvimento saudável do indivíduo e da sociedade; atenta para grupos marginalizados que dependem das escolas; defende o retorno seguro das crianças às escolas quando a transmissão local estiver sob controle; e o aumento dos orçamentos destinados à educação, alfabetização e infraestrutura digital.

Documento que aborda as principais ações preventivas e de controle do COVID-19 nas escolas.

No documento, constam orientações claras e específicas para o retorno à escola, como: manter indivíduos doentes ou com suspeita de contaminação isolados; reforçar hábitos de higiene pessoal e do local, desinfetando superfícies e incentivando a lavagem regular das mãos; promover e garantir o distanciamento social; manter a comunidade escolar informada sobre a doença do novo coronavírus; não promover aglomeração de pessoas; usar estratégias de ensino online; e apoiar populações vulneráveis que dependem da escola.

O documento ressalta que a proporção de crianças infectadas por COVID-19 é pequena (menor que 5\%); quando diagnosticadas, têm menor probabilidade de serem hospitalizadas ou apresentar quadro grave, podendo apenas ter sintomas leves ou não apresentarem sintomas. Quanto ao ambiente escolar, trata da dificuldade em detectar casos em crianças pela falta de sintomas, mas reforça que houve poucos surtos significativos. Defende que, tomadas as devidas medidas de higiene e distanciamento apropriadas, as escolas provavelmente não se tornarão ambientes de propagação. Apesar das evidências conflitantes, a reabertura de escolas não foi associada a aumentos significativos na transmissão comunitária. Adicionalmente, o impacto socioemocional é um dos pontos fortes de justificativa para o retorno.

Como consequência da medida, mais de 70\% da população estudantil mundial foi afetada, representando mais de 900 milhões de estudantes. Só no Brasil, são mais de 50 milhões de afetados.

Apresentar dados
Suspensão das aulas e respostas à COVID-19 (UNESCO) $)^{36}$
Outubro/2020 do impacto do COVID-19 na educação através do monitoramento mundial do fechamento de escolas. 
1 Professora titular, Faculdade de Psicologia e Programa de Pós-Graduação em Psicologia (Cognição Humana), Pontifícia Universidade Católica do Rio Grande do Sul (PUCRS), Porto Alegre, RS. Presidente, Sociedade Brasileira de Neuropsicologia (SBNp), São Paulo, SP. ${ }^{2}$ Psicóloga pela PUCRS, Porto Alegre, RS. Pós-graduanda em Psicologia do Desenvolvimento e da Aprendizagem, PUCRS, Porto Alegre, RS. ${ }^{3}$ Graduação em Medicina, Universidade Federal do Rio Grande do Sul (UFRGS), Porto Alegre, RS. Residência Médica em Otorrinolaringologia, Hospital São Lucas da PUCRS, Porto Alegre, RS. Fellowship em Otorrinolaringologia Pediátrica, Hospital de Clínicas de Porto Alegre(HCPA), Porto Alegre, RS. Mestre em Saúde da Criança e Adolescente pela UFRGS, Porto Alegre, RS.

de aprendizagem podem deixar marcas difíceis de apagar, com gaps de desenvolvimento social e acadêmico, com prejuízos ainda desconhecidos.

Outras manifestações de cunho científico a favor ou contrárias ao presente posicionamento baseado nas principais e mais recentes publicações acerca do tema são encorajadas. Tais manifestações, entretanto, precisam ser alicerçadas em evidências de pesquisas, não meramente em notícias cujas manchetes tendem a estimular condições emocionais dominadas por medo e estresse. Deve-se, ainda, salientar que a escola vai além do ambiente que provém conhecimento formal; é um espaço para se relacionar, desenvolver habilidades socioemocionais e, para os mais vulneráveis, as escolas também servem como garantia de uma alimentação saudável ${ }^{1,3}$.

Estudos demonstram como as desigualdades entre diferentes níveis socioeconômicos podem impactar na aprendizagem, com pesquisas que constatam as lacunas em habilidades matemáticas e de alfabetização entre crianças dos extremos níveis socioeconômicos (alto e baixo) em períodos de férias, isto é, crianças mais socioeconomicamente desprovidas acabam sofrendo mais impacto pela falta de estimulação que a escola prevê do que as crianças de lares de maior poder aquisitivo ${ }^{3}$, possivelmente pelas oportunidades de estimulação em casa.

Considerando-se que a pandemia vem abrindo cada vez mais as lacunas entre os diferentes níveis socioeconômicos, há necessidade de intervenções políticas e de legislação, a fim de fornecer apoio e de prevenir o aprofundamento e ampliação da desigualdade social, que, por consequência, corresponde a uma desigualdade maior do que a anteriormente caracterizada no processo de aprendizagem. Sem essas estratégias, a atual crise de saúde pode se transformar em uma crise de consequências duradouras para as crianças de famílias de baixa renda ${ }^{3}$.

Por fim, destaca-se que há relatos em grupos menores de ausência de dificuldades cognitivas, socioemocionais ou de aprendizagem, com a principal associação do fator de maior convivência familiar na ausência de conflitos ou de vulnerabilidades. Mais estudos devem ser conduzidos para que diferentes evoluções possam ser mapeadas e a relação entre fatores negativos e positivos possa ser aprofundada, com aplicabilidades para o desenvolvimento de programas de intervenção clínica e educacional nas escolas com professores, pais e alunos.

\section{Agradecimentos}

Agradecemos aos alunos de iniciação científica Valentina Fiorioli e Deivid de Franceschini Felizardo, que auxiliaram na transcrição de partes das aulas abertas (lives) para que o máximo de sua reprodução na presente escrita científica pudesse ser abarcado, assim como à mestranda Ana Carolina Rodrigues, por seu auxílio na organização inicial do artigo.

Artigo submetido em 30/10/2020, aceito em 30/10/2020. Os autores informam não haver conflitos de interesse associados à publicação deste artigo.

Fontes de financiamento inexistentes.

Correspondência: Rochele Paz Fonseca, Avenida Ipiranga, 6681, Prédio 11, Sala 940, CEP 90619-900, Porto Alegre, RS. E-mail: rochele.fonseca@gmail.com

\section{Referências}

1. Macartney K, Quinn HE, Pillsburry AJ, Epi MA, Koirala A, Deng L, et al. Transmission of SARSCoV-2 in Australian educational settings: a prospective cohort study. Lancet Child Adolesc Health. 2020;4:807-16.

2. European Centre for Disease Prevention and Control (ECDC). COVID-19 in children and the role of school settings in COVID-19 transmission [Internet]. 2020 Aug 6 [cited 2020 Nov 9]. www. ecdc.europa.eu/en/publications-data/childrenand-school-settings-covid-19-transmission

3. Van-Lancker W, Parolin Z. COVID-19, school closures, and child poverty: a social crisis in the making. Lancet Public Health. 2020;5:e243-4.

4. Rajmil L. Role of children in the transmission of the COVID-19 pandemic: a rapid scoping review. BMJ Paediatrics Open. 2020;4:e000722.

5. Lee B, Raszka WV. COVID-19 transmission and children: the child is not to blame. Pediatrics. 2020;146:e2020004879.

6. Li X, Xu W, Dozier M, He Y, Kirolos A, Theodoratou $E$, et al. The role of children in transmission of SARS-CoV-2: a rapid review. J Glob Health. 2020;10:011101. 

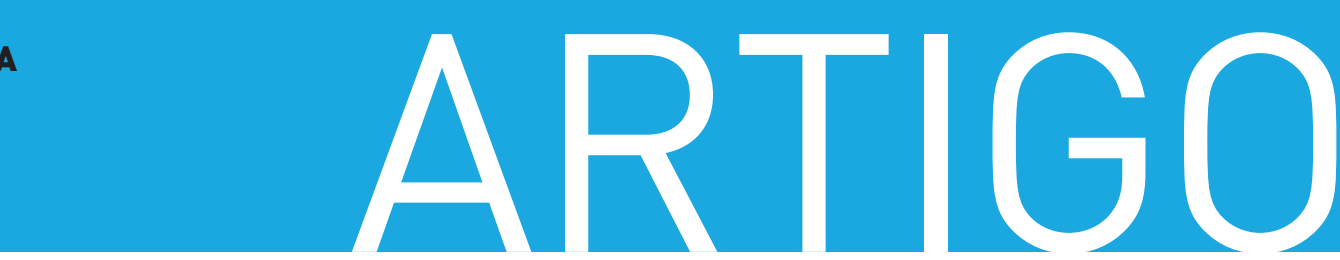

7. Goldstein E, Lipsitch M, Cevik M. On the effect of age on the transmission of SARS-CoV-2 in households, schools and the community. Version 2. medRxiv. 2020; Preprint. 2020 Jul 24 [revised 2020 Jul 28].

8. Viner RM, Mytton OT, Bonell C, MelendezTorres GJ, Ward J, Hudson L, et al. Susceptibility to SARS-CoV-2 infection among children and adolescents compared with adults: a systematic review and meta- analysis. JAMA Pediatr. 2020 Sep 25;e204573. doi: 10.1001/ jamapediatrics.2020.4573. Online ahead of print.

9. Safadi MAP. As características intrigantes da COVID-19 em crianças e seu impacto na pandemia. J Pediatr (Rio J). 2020;96:265-8.

10. Fundação Oswaldo Cruz (FIOCRUZ), Instituto Nacional de Saúde da Mulher, da Criança e do Adolescente Fernandes Figueira, Sistema Único de saúde (SUS). COVID-19 e saúde da criança e do adolescente [Internet]. 2020 Aug 11 [cited 2020 Nov 9]. portaldeboaspraticas.iff.fiocruz. br/atencao-crianca/covid-19-saude-crianca-eadolescente/

11. Rice K, Wynne B, Martin V, Ackland GJ. Effect of school closures on mortality from coronavirus disease 2019: old and new predictions. BMJ. 2020;371:m3588.

12. Ladhani, S. Prospective active national surveillance of preschools and primary schools for SARS-CoV-2 infection and transmission in England, June 2020 (sKIDs COVID-19 surveillance in school KIDs) [Internet]. 2020 Sep 1 [cited 2020 Nov 9]. assets. publishing.service.gov.uk/government/uploads/ system/uploads/attachment_data/file/914700/ sKIDs_Phase1Report_01sep2020.pdf

13. Danis K, Epaulard O, Bénet T, Gaymard A, Campoy S, Botelho-Nevers E, et al. Cluster of coronavirus disease 2019 (COVID-19) in the French Alps, February 2020. Clin Infect Dis. 2020;71:825-32.

14. Wu Q, Xing Y, Shi L, Li W, Gao Y, Pan S, et al. Coinfection and other clinical characteristics of COVID-19 in children. Pediatrics. 2020;146:e20200961.

15. Auger KA, Shah SS, Richardson T, Hartley D, Hall M, Warniment A, et al. Association between statewide school closure and COVID-19 incidence and mortality in the US. JAMA. 2020;324:85970.

16. Donohue JM, Miller E. COVID-19 and school closures. JAMA. 2020;324:845-7.

17. Viner RM, Russell SJ, Croker H, Packer J, Ward J, Stansfield C, et al. School closure and management practices during coronavirus outbreaks including COVID-19: a rapid systematic review. Lancet Child Adolesc Health. 2020;4:397-404.

18. Esposito S, Principi N. School closure during the coronavirus disease 2019 (COVID-19) pandemic: an effective intervention at the global level? JAMA Pediatr. 2020;174:921-2.

19. Dubey S, Biswas P, Ghosh R, Chatterjee S, Dubey MJ, Chatterjee S, et al. Psychosocial impact of COVID-19. Diabetes Metab Syndr. 2020;14:77988.

20. Malloy-Diniz LF, Costa DS, Loureiro F, Moreira L, Silveira BKS, Sadi HM, et al. Saúde mental na pandemia de COVID-19: considerações práticas multidisciplinares sobre cognição, emoção e comportamento. Debates Psiquiatr. 2020;2:4668.

21. Guimarães TT, dos Santos HMB, Sanctos RTM. Physical inactivity, chronic diseases, immunity and COVID-19. Rev Bras Med Esporte. 2020;26:37881.

22. Antunes J. Estresse e doença: o que diz a evidência? Psic Saude Doenças. 2019;20:590603.

23. Marques-Deak A, Sternberg E. [Psychoneuroimmunology--the relation between the central nervous system and the immune system]. Braz J Psychiatry. 2004;26:143-4.

24. Maia AC. Emoções e sistema imunológico: um olhar sobre a psiconeuroimunologia. Psicol Teor Prat. 2002;2:207-25.

25. Williams PG, Lerner MA; Council on Early Childhood; Council on School Health. School readiness. Pediatrics. 2019;144:e20191766.

26. Aunio P, Korhonen J, Ragpot L, Törmänen M, Mononen R, Henning E. Multi-factorial approach to early numeracy--the effects of cognitive skills, language factors and kindergarten attendance on 
1 Professora titular, Faculdade de Psicologia e Programa de Pós-Graduação em Psicologia (Cognição Humana), Pontifícia Universidade Católica do Rio Grande do Sul (PUCRS), Porto Alegre, RS. Presidente, Sociedade Brasileira de Neuropsicologia (SBNp), São Paulo, SP. ${ }^{2}$ Psicóloga pela PUCRS, Porto Alegre, RS. Pós-graduanda em Psicologia do Desenvolvimento e da Aprendizagem, PUCRS, Porto Alegre, RS. ${ }^{3}$ Graduação em Medicina, Universidade Federal do Rio Grande do Sul (UFRGS), Porto Alegre, RS. Residência Médica em Otorrinolaringologia, Hospital São Lucas da PUCRS, Porto Alegre, RS. Fellowship em Otorrinolaringologia Pediátrica, Hospital de Clínicas de Porto Alegre(HCPA), Porto Alegre, RS. Mestre em Saúde da Criança e Adolescente pela UFRGS, Porto Alegre, RS

early numeracy performance of South African first graders. Int J Educ Res. 2019;97;65-76.

27. Larsen B, Luna B. Adolescence as a neurobiological critical period for the development of higher-order cognition. Neurosci Biobehav Rev. 2018;94:17995.

28. Morgan K, Melendez-Torres GJ, Bond A, Hawkins J, Hewitt G, Murphy S, et al. Socio-economic inequalities in adolescent summer holiday experiences, and mental wellbeing on return to school: analysis of the school health research network/health behaviour in school-aged children survey in Wales. Int J Environ Res Public Health. 2019;16:1107.

29. Guio AC, Gordon D, Marlier E, Najera H, Pomati M. Towards an EU measure of child deprivation. Child Indic Res. 2018;11:835-60.

30. Ostrosky-Solis F, Ramirez M, Ardila A. Effects of culture and education on neuropsychological testing: a preliminary study with indigenous and nonindigenous population. Appl Neuropsychol. 2004;11:186-95.

31. Parente MAMP, Scherer LC, Zimmermann N, Fonseca RP. Evidências do papel da escolaridade na organização cerebral. Neuropsicol Latinoam. 2009:1:72-80.

32. Clemens $V$, Deschamps $P$, Fegert JM, Anagnostopoulos D, Bailey S, Doyle $M$, et al. Potential effects of "social" distancing measures and school lockdown on child and adolescent mental health. Eur Child Adolesc Psychiatry. 2020;29:739-42.

33. Fegert JM, Vitiello B, Plener PL, Clemens V. Challenges and burden of the Coronavirus 2019 (COVID-19) pandemic for child and adolescent mental health: a narrative review to highlight clinical and research needs in the acute phase and the long return to normality. Child Adolesc Psychiatry Ment Health. 2020;14:20.

34. Brown SM, Doom JR, Lechuga-Peña S, Watamura SE, Koppels T. Stress and parenting during the global COVID-19 pandemic. Child Abuse Negl. 2020 Aug 20;104699. doi: 10.1016/j. chiabu.2020.104699. Online ahead of print.

35. Brooks SK, Webster RK, Smith LE, Woodland L, Wessely S, Greenberg N, et al. The psychological impact of quarantine and how to reduce it: rapid review of the evidence. Lancet. 2020;395;91220.

36. UNESCO. Suspensão das aulas e resposta à COVID-19 [internet]. 2020 [cited 2020 Nov 9]. pt.unesco.org/covid19/educationresponse

37. UNICEF. Key messages and actions for COVID-19 prevention and control in schools [Internet]. 2020 Mar [cited 2020 Nov 9]. www.who.int/docs/ default-source/coronaviruse/key-messages-andactions-for-covid-19-prevention-and-control-inschools-march-2020.pdf?sfvrsn=baf81d52_4

38. World Health Organization (WHO). Advice on the use of masks for children in the community in the context of COVID-19: annex to the Advice on the use of masks in the context of COVID-19 [Internet]. 2020 Aug 21 [cited 2020 Nov 9]. www. who.int/publications/i/item/WHO-2019-nCoVIPC_Masks-Children-2020.1

39. United Nations (UN). Launch of the policy brief: education during COVID-19 and beyond - "The future of education ishere" [Internet].2020 [cited 2020 Nov 9]. www.un.org/en/coronavirus/ future-education-here 